\title{
Off-set Filtering Effect in SOA Based Optical Access Network
}

\author{
Eszter UDVARY \\ Dept. of Broadband Infocommunications and Electromagnetic Theory, \\ Budapest University of Technology and Economics, 3 Muegyetem rakpart, 1111 Budapest, Hungary \\ udvary@mht.bme.hu
}

Manuscript received September 25, 2015

\begin{abstract}
In-line and reflective semiconductor optical amplifiers (SOA and RSOA) can be effectively utilized as external optical intensity modulators. However the limited modulation bandwidth affects the application possibilities, because next generation access networks desire high transmission speed. Bandwidth enhancement can be achieved by means of optical equalization thanks to the chirping characteristics of semiconductor optical amplifier. The limited modulation transfer function can be combined with the converted phase modulation transfer function. Investigation of optical filter with optimal slope is a crucial challenge in this approach. The presented simulations describe the slope dependence of the modulation band. The model can take into account the different type of realizable optical filters. However there is a trade-off between modulation efficiency and modulation bandwidth due to the extra insertion loss of the set-off optical filter. In the paper theoretical and simulation investigations are presented with the result of improved modulation bandwidth.
\end{abstract}

\section{Keywords}

Semiconductor Optical Amplifier, modulation bandwidth, frequency discriminator, optical filter, chirp parameter

\section{Introduction}

In-line and reflective semiconductor optical amplifiers (SOAs and RSOAs) turn out to be key components for $60 \mathrm{GHz}$ Radio over Fibre systems (60G-RoF) and new generation, wavelength division multiplexed passive optical networks (WDM-PON) since they allow for directlymodulated colorless transceivers.

RSOAs and SOAs have demonstrated their multifunctional capability by combining optical amplification with either modulation [1], gating, photodetection [2], dispersion compensation [3], linearization [4], commutation, wavelength conversion, signal regeneration, etc. It is a semiconductor based, small size, low cost, current driven device. (R)SOA-modulator requires low modulation power, and the detected electrical power is high because of the optical gain in contrary to the optical insertion loss of other modulators. However modulation bandwidth of RSOA is usually limited to around $1 \div 3 \mathrm{GHz}$. Increasing the modulation bandwidth of RSOA is still a challenge.

The remainder of the paper is organized as follows. Section 2 presents calculation and experimental results, which show the effects of different internal device parameters and environmental conditions. Section 3 provides some background on novel, off-set filtering technique. Section 4 describes the simulation environment. Finally, Section 5 presents the simulation results.

\section{Optimized Device and System Parameters}

The frequency response of a semiconductor optical amplifier has a smooth roll-off with no relaxation oscillation peak, while its modulation has a good linearity similar to the laser diode. The modulation bandwidth of SOA or RSOA is limited by the speed at which the carrier density can be changed. This is usually determined by the lifetime of the carriers in the active layer. The typical value of carrier lifetime is about 300 ps and the value of the bandwidth is in the gigahertz range. It can be improved by saturation and propagation effects. Under the influence of higher optical intensities the stimulated lifetime will dominate and the bandwidth extended to $100 \mathrm{GHz}$ regime.

So, carrier lifetime is mainly governed by the emission rate [5]. Based on it, the operational and environmental parameters can be optimized from the view point of the modulation speed. These methods were investigated by simulation and experimental work.

\subsection{Calculation Model}

The applied model used the wave and rate equations. An optical field which propagates along a travelling wave semiconductor optical amplifier was considered in the 
model. The interaction of light with carriers in the SOA is governed by the carrier rate and field propagation wave equations. The amplifier's output power is calculated by solving numerically the coupled rate and wave equations [5]. First the operating point is calculated by a steady state consideration. Next a change in the carrier density around the mean value was considered due to a change in the injected signal and the differential equation was obtained. The associated change in the stimulated and spontaneous emissions can be calculated.

$$
\begin{aligned}
& \frac{d N}{d t}=\frac{I}{q A}-R_{\mathrm{sp}}(N)-v_{\mathrm{g}} \Gamma g_{\mathrm{m}} S, \\
& \frac{d S}{d z}=\left(\Gamma g_{\mathrm{m}}-\alpha\right) S
\end{aligned}
$$

where $N$ is the carrier density, $I$ is the injection current, $q$ is the electron charge, $A$ is the volume of active layer, $R_{\mathrm{sp}}(N)$ is the spontaneous recombination rate, $v_{\mathrm{g}}$ is group velocity, $\Gamma$ is the optical confinement factor, $g_{\mathrm{m}}$ is the material gain, $\alpha$ is the internal loss, $S$ is the photon flux density, $t$ is the local time, $z$ is the spatial coordinate along the amplifier.

In case of modulation, the current is divided into DC and radiofrequency modulation parts. So carrier and photon densities include DC and modulation parts, too.

$$
\begin{aligned}
& I=I_{\mathrm{DC}}+\Delta I \cdot \exp (j \omega t), \\
& S(t, z)=S_{\mathrm{DC}}(z)+\Delta S(z) \cdot \exp (j \omega t), \\
& N(t, z)=N_{\mathrm{DC}}+\Delta N(z) \cdot \exp (j \omega t) .
\end{aligned}
$$

So, the modulation amplitude of the photon density can be calculated at the output of the device.

$$
\begin{aligned}
\frac{d \Delta S}{d z}= & \left(g_{\mathrm{sat}}(z)-\alpha\right) \cdot \Delta S(z)+\Gamma \cdot a \cdot \Delta N(z) \cdot S_{\mathrm{DC}}(z)= \\
& =\left(g_{\mathrm{sat}}(z)-\alpha\right) \cdot \Delta S(z)+ \\
& +\Gamma \cdot a \cdot \frac{\frac{\tau_{\mathrm{s}}}{e \cdot V} \cdot \Delta I(z)-\frac{v_{\mathrm{g}} \cdot \tau_{\mathrm{s}} \cdot g_{\mathrm{sat}}(z)}{\Gamma} \cdot \Delta S(z)}{1+j \cdot \omega \cdot \tau_{\mathrm{s}}+\frac{S_{\mathrm{DC}}(z)}{S_{\mathrm{sat}}}} \cdot S_{\mathrm{DC}}(z)
\end{aligned}
$$

where $g_{\text {sat }}$ is the saturated gain, $S_{\text {sat }}$ is the saturated photon flux density, $\tau_{\mathrm{s}}$ is the carrier lifetime, $a$ is the differential gain, $j$ is the imaginary unit.

The carrier density is non-uniform along the SOA active region. So the device is divided into many longitudinal sections and the applied model assumes both uniform carrier and photon densities in each section. The sectioned amplifier cavity is used to account for longitudinal effects; and the spatial variation of the material gain and other parameters of the SOA can be captured. Consequently, the propagation effect can be simulated by this method. The suitable adjustment of the model parameters enables the simulation of both in-line and reflective amplifiers. However the effect of spatial dependence in an in-line device is more significant than in a reflective device.

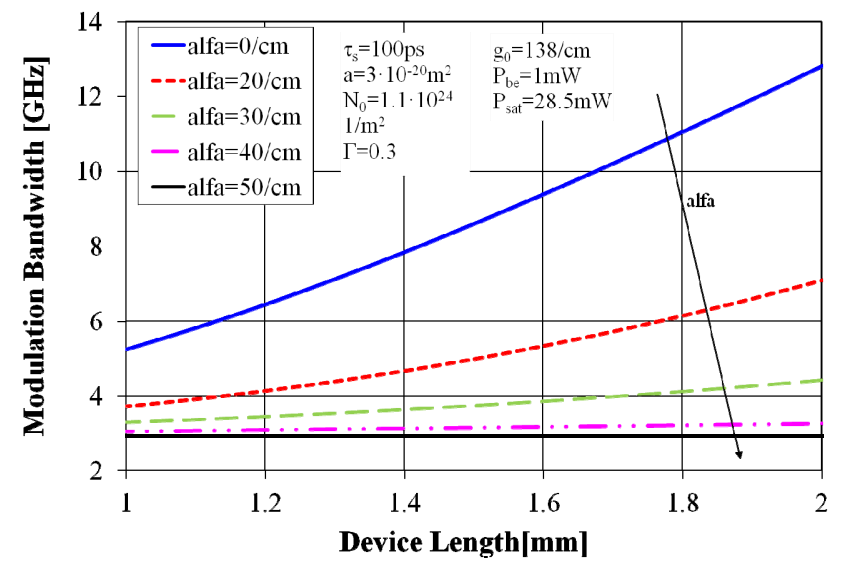

Fig. 1. Simulated bandwidth versus device length with different internal attenuations.

$3-\mathrm{dB}$ and $10-\mathrm{dB}$ modulation bandwidths can be detected from the simulated transfer function. The equation suggests that an increased bandwidth will be obtained with increased current, input optical power, differential gain and confinement factor. So, the length of the RSOA can be enlarged to increase photon density, hence reducing carrier lifetime. The bandwidth increases versus device length. The slope is higher in case of lower internal loss. On the other hand, the bandwidth decreases versus internal loss. Namely it is improved by higher optical intensities. The modulation bandwidth is extended if we take into account propagation effect.

The simulation results in Fig. 1. show that bandwidth is improved and a resonance can be observed as device length increases. On the other hand, lower attenuation, namely higher intensity also improves the bandwidth.

\subsection{Experimental Validation}

The frequency response of the RSOA can be measured by modulating the carrier density. During the experimental work the RSOA-modulator under test was driven by the sum of bias current and sinusoidal modulation radio frequency signal via a bias tee. The frequency of the modulation signal is swept, and the detected electrical signal is registered at the electrical output. The amplitude and shape of the transfer function mainly depend on input optical power and bias current.

Figure 2 shows the relative modulation bandwidth versus bias current as a function of the input optical power. The improvement of the bandwidth is approximately directly proportional to the bias current. The slope of the curve is higher in the saturation regime, practically in case of higher input power. So, the effect is more significant in the saturated regime.

In Fig. 3 the relative bandwidth versus input optical power curve is plotted. The modulation bandwidth is constant in the unsaturated regime. However it can be 


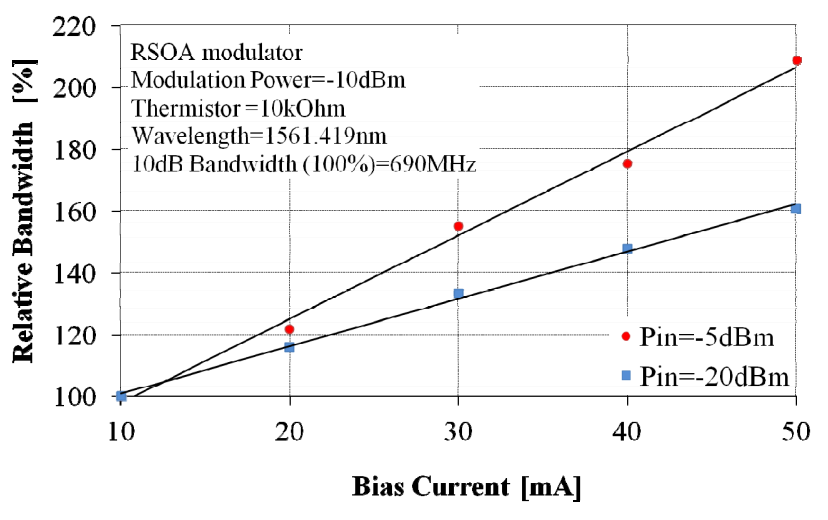

Fig. 2. Relative bandwidth versus bias current.

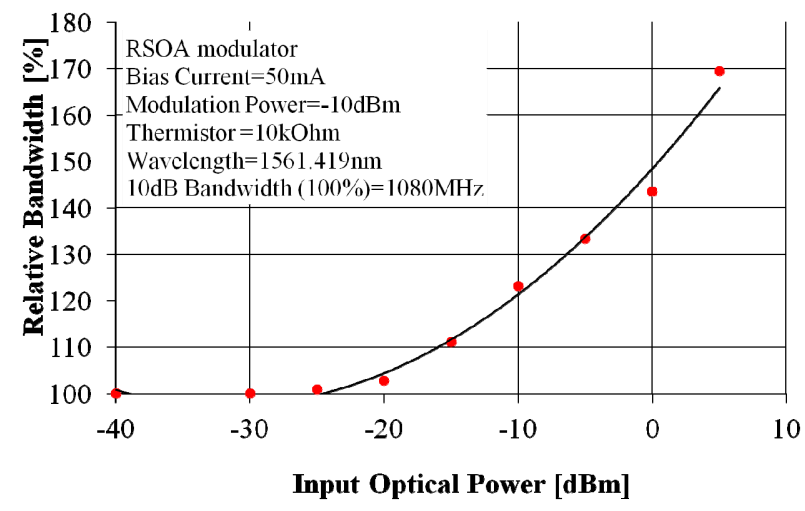

Fig. 3. Relative bandwidth versus input optical power.

extended by about 70 percent compared with the unsaturated value in the saturated regime.

The simulation and experimental investigation confirmed the effects of device and system parameters. The modulation speed can be improved by proper selection of the parameters. However, the realized bandwidth is not enough for future, high speed applications. Further bandwidth improvement methods are needed.

\section{Phase-to-Intensity Conversion}

Bandwidth enhancement can be also achieved by means of optical equalization thanks to the chirping characteristics of the device [6]. Direct modulation of a (R)SOA produces optical signals modulated both in amplitude and phase [1]. The amplitude modulation is received by direct detection, when the effective modulation bandwidth is limited by the frequency response of the (R)SOA. On the other hand, phase modulation is disregarded. The main idea is, that the phase modulated information can be converted to amplitude modulation. The intensity modulation is low pass type. If the converted phase modulation causes high pass filtering, the bandwidth of the whole transfer function will be enhanced. The conversion compensates the limitation of amplitude modulation bandwidth and effectively extends the overall frequency response of the system. The method combines the low-pass response of the (R)SOA and the high-pass response added by the converted phase modulation.

The phase-to-intensity (PM-IM) modulation conversion has been used in the microwave techniques for a long time. It is also useful for phase noise measurement in optical communication, where an optical frequency discriminator is utilized to transfer the fluctuations of the optical frequency into intensity variations.

The frequency discriminator is characterized by its sensitivity or discrimination slope ( $D$ in [W/Hz]) [11].

$$
D=\frac{\Delta \text { Power }}{\Delta \text { Phase }}
$$

where $\Delta$ Power is the intensity variation and $\Delta$ Phase is the phase variation. It shows the ability to convert phase fluctuations of the input signal into variations of the output power. So, higher discriminator slope implements better conversion and linear transmission versus frequency profile is desired.

Phase-to-intensity conversion can be realized by signal propagation over an optical fiber, but it is not effective. Other solution is the application of an optical frequency discriminator, which can be realized by frequency selective attenuation, practically by an optical filter. The MachZehnder interferometer was first suggested, but it has high nonlinearity and it creates high distortion components. Several other solutions were proposed like Fabry-Perot interferometer, fiber bragg grating and ring resonators. Anyhow, linear demodulation could be accomplished by a linear transfer function. So high-order filter could be used to implement it.

So, an optical filter with a suitable slope provides such PM-IM conversion. Any filter (low-pass, high-pass, band-pass) can provide this condition. In the used optical spectrum this function can be realized by applying an optical band pass filter detuned from the carrier frequency of the optical signal. This approach is called set-off filtering [7]. And proper set-off filtering could improve the transmission performance.

The optimal transfer function of the optical filter, including detuning, optical bandwidth, transitions and type of the optical filter have to be chosen carefully. Moreover, the parameters of the optical filter are not independent. The narrower bandwidth of the filter usually induces a steeper slope. On the other hand, wide tolerance to the wavelength detuning is important, because it removes the need for electronic equalization. Set-off filtering induces extra loss and the PM-IM conversion demands steep slope. The trade-off between the minimal extra loss and optimal slope of the curve is important. Comparison of positive and negative frequency deviations at the rising and falling edges is also relevant. Both of them can be exploited; but for positive frequency detuning, the signal lower frequencies are reduced; while for negative detuning the signal higher frequency components are limited. 


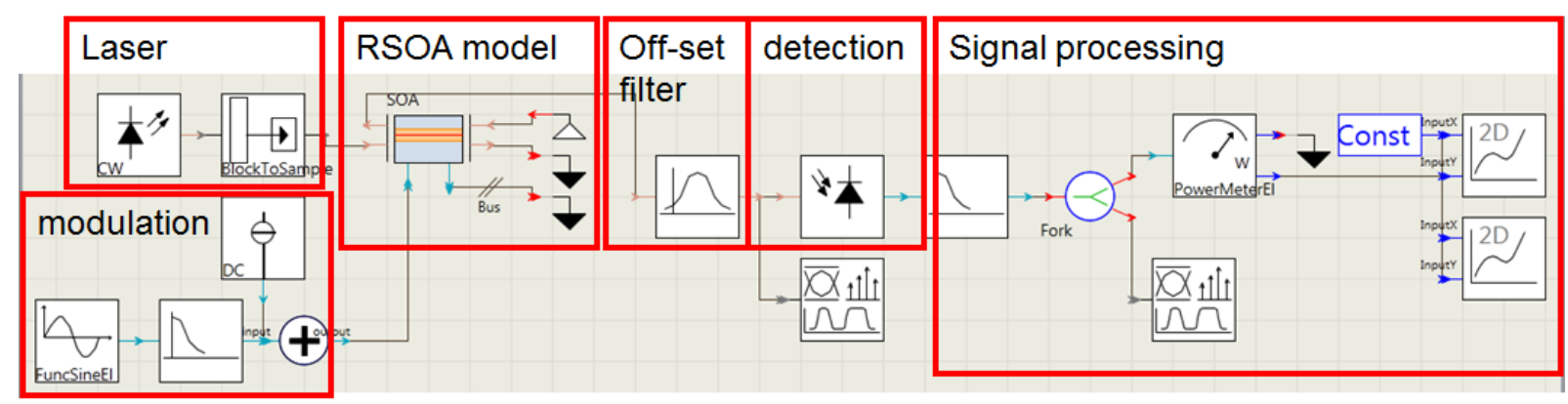

Fig. 4. Simplified block diagram.

\section{Simulation Setup}

The simulations were implemented in Virtual Photonics Transmission Maker 9.0 simulation environment. The simulation set-up followed the typical architecture of the optical access network. The continuous wave optical signal is generated by a laser diode. Due to the chirp effect, it is intensity- and phase-modulated by RSOA. The intensityand phase-modulated and reflected signal is separated from the incident optical signal by an optical circulator. The signal is sent through the set-off optical filter and it is detected by a high speed photodiode. Figure 4 presents the simplified block diagram of the simulation system. The blocks are described in greater details in Sec. 4.1-4.4.

\subsection{Amplifier Chirp}

The optical gain of a laser amplifier is affected in both magnitude and phase via the modulation of the complex refractive index caused by the electron density modulation. In other words, direct current modulation is associated with a carrier density variation, which causes a chirp accompanying the intensity modulated signal. The adiabatic chirp refers to a shift between the emissions frequencies of the two signal levels; it is proportional with the optical power. The transient chirp appears at the rising and falling edges of the modulated signal and it depends on the first time derivative of the emitted power. Hence, its time duration and magnitude are determined by the modulation bandwidth of the device.

Chirp effect can be modeled using Linewidth Enhancement Factor approximation. The chirp parameter is the ratio of the amplitude modulation and the phase modulation. It is expressed as

$$
L E F_{\text {unsat }}=-\frac{4 \cdot \pi}{\lambda} \cdot \frac{\partial n / \partial N}{\partial g / \partial N}
$$

where $\lambda$ is the lasing wavelength, $N$ is the carrier density, $n$ is the refractive index and $g$ is differential gain.

Measurements can be found in the literature and have shown that $L E F$ changes depending on several parameters such as bias current, wavelength and input optical power. In the unsaturated region the $L E F$ value ranges from 2 to 7 for GaAs and GaInAsP bulk devices and from 1.5 to 2 for quantum well structures [8]. However, the chirping parameter which is positive for light sources and unsaturated optical amplifiers is negative for saturated amplifiers [9]. The saturated chirp parameter can be calculated as

$$
L E F=L E F_{\text {unsat }} \cdot \frac{\mathrm{d} G / \mathrm{d} P_{\text {in }}}{1+\left(\mathrm{d} G / \mathrm{d} P_{\text {in }}\right)}
$$

where $G$ is the optical gain in $\mathrm{dB}$ and $P_{\text {in }}$ is the input optical power in $\mathrm{dBm}$.

The gain variation and the phase variation of the device can be expressed as

$$
\begin{aligned}
& I M=\frac{\Delta G}{G}=\frac{\mathrm{d} g}{\mathrm{~d} N} L \Delta N, \\
& P M=\Delta \phi=\frac{L E F}{2} \frac{\mathrm{d} g}{\mathrm{~d} N} L \Delta N \Delta G=q \Gamma g \Delta N L
\end{aligned}
$$

where $\Delta G$ is the optical gain variation, $\Delta \Phi$ is the phase variation of the output optical signal, $N$ is the carrier density, $q$ is the electron charge, $\Gamma$ is the light confinement factor, $L$ is the device length.

\subsection{Phase-to-Intensity Conversion}

The phase-to-intensity conversion is realized by frequency discriminator. The discriminator has a frequency dependent amplitude transfer function. The slope of the function converts the variation in the optical frequency into variation in the amplitude. Sidebands of a frequency modulated signal possess certain amplitude and phase relationships among themselves such that the envelope of the signal is independent of time. A discriminator works by modifying these phase and amplitude relationships such that the amplitude of the envelope of the resultant signal fluctuates in the same manner versus time as the instantaneous frequency of the original signal did. It suggests that functions with larger slopes will have better conversion efficiency. And the IM signal is distorted by the nonlinearities.

Practical optical filters are coherent, because of the long coherence lengths of lasers. So, it is analyzed by electric field amplitude and phase. The chirped optical signal that is phase- and intensity-modulated by sinusoidal tone can be represented by the electrical field: 


$$
e(t)=\sqrt{2} P_{\mathrm{opt}} \sqrt{1+m \cos (\omega t+\varphi) \cos \left(\omega_{\mathrm{c}} t+\beta \sin \omega t\right)}
$$

where $P_{\text {opt }}$ is the average optical power, $\omega_{\mathrm{c}}$ is the angular frequency of the optical carrier, $\beta$ is the angle modulation depth, $\omega$ is the modulation angular frequency, $m$ represents the intensity modulation depth and $\varphi$ is the phase difference between the phase and intensity modulations.

Applying the Jacobi-Anger expansion the electrical field at the output of the filter can be expressed as an infinite weighted sum over sidebands. At the output of the photodetector the signal current at the modulation frequency is [10]:

$$
i=R P_{\text {opt }} \operatorname{Re}\{[\beta X+0.5 m Y \exp (j \phi)] \exp (j \omega \varphi\}
$$

where $R$ is the responsivity of the photodetector, $j$ is the imaginary unit, $X$ and $Y$ are complex constants of the filter and $\operatorname{Re}\{\}$ means the real part.

Consequently, the optical signal at the output of the detuned filter can be written:

$$
\begin{aligned}
\Delta P_{\text {out }} & =\text { att } \Delta P_{\text {in }}+\text { slope } \Delta f= \\
& =\text { att } \Delta P_{\text {in }}+\text { slope } \frac{1}{2 \pi} \frac{\mathrm{d} \Delta \Phi}{\mathrm{d} t}
\end{aligned}
$$

where att is the attenuation of the filter and slope is the slope of the filter, $P_{\text {in }}$ is the optical power at filter input, $\Delta f$ is the frequency variation, which is the derivation of phase variation.

$$
\Delta P_{\text {out }}=a t t \cdot P_{\mathrm{LD}} \cdot \Delta G \cdot G+\text { slope } \cdot \Delta G \cdot G \cdot \frac{L E F}{4 \cdot \pi} \frac{\mathrm{d} \Delta G}{\mathrm{~d} t}
$$

In terms of Fourier transform it can be expressed as

$$
P_{\text {out }}(j \omega)=P_{\mathrm{LD}} \Delta G(\omega) G\left[\text { att }+ \text { slope } \frac{L E F}{4 \pi P_{\mathrm{LD}}}(j \omega)\right]
$$

The term (jw) means a high pass contribution, which represents the phase-to-intensity converted term. It is contrast with the low lass response in the intensity modulated term.

\subsection{Optical Filters}

Three filter types were investigated, which had different transfer functions. The parameters of the investigated optical filters were limited by practical considerations. In fact, a physically realizable optical filter must be chosen.

The rectangular filter passes the signal unaltered in the pass band and reduces the power by a given attenuation outside this range. The transition is ideal and the phase response is constant. Hence this filter is not applicable for PM-AM conversion. The trapezoidal filter provides similar transmission as the rectangular one, but with some transition band. It is an ideal filter within pass band, an arbitrary attenuation at stop band and linearly increasing attenuation at transition from the pass band to stop band. Because of its ideal feature simulations were carried out to examine the filter roles and the required bandwidth, set-off frequency and slope of the filter. In the simulation the filter's attenuation in the stop band was $50 \mathrm{~dB}$, the filter's bandwidth was set $20 \mathrm{GHz}$. The frequency detuning between the $\mathrm{CW}$ seeding optical frequency and the optical filter centre frequency was swept from $-30 \mathrm{GHz}$ to $30 \mathrm{GHz}$. In the other words, both positive and negative set-offs were investigated. On the other hand, 5, 2.5 and $1 \mathrm{~dB} / \mathrm{GHz}$ filter slopes were studied.

Butterworth Filter is a real optical filter type, which has maximally flat band pass. It has smaller slope between the pass band and the stop band, than the trapezoidal filter. So it is more sensitive for the value of the set-off frequency. This filter has the flattest possible pass-band magnitude response. Attenuation is $-3 \mathrm{~dB}$ at the design cut-off frequency. Attenuation beyond the cut-off frequency is a moderately steep $-20 \mathrm{~dB} /$ decade/pole. The pulse response of the Butterworth filter has moderate overshoot and ringing. The simulated filter was the fifth order Butterworth filter and its centre frequency was also set-off by $23 \mathrm{GHz}$ with a bandwidth of $20 \mathrm{GHz}$. In the effective optical band its slope was similar to the trapezoid slope (Fig. 5).

Next, an optical filter with Gaussian amplitude and zero phase of the transfer function was investigated. Application of Gaussian shaped optical filter is more realistic, because it is commercially available. For example Array Waveguide Grating (AWG) has near Gaussian shape. The simulated filter was 1.5 order Gaussian type and the centre frequency was also set-off by $23 \mathrm{GHz}$ with a bandwidth of $20 \mathrm{GHz}$. In the used optical band the slope was similar to the trapezoid slope (Fig. 5.).

\subsection{Chromatic Dispersion}

The signal shape changes because of the dispersion as the signal propagates over various fiber distances. Chromatic dispersion significantly limits the modulation bandwidth in case of long optical link, because the different spectral components travel with different velocities and phase difference is observed between the components. The frequency transfer function of the optical link can be written:

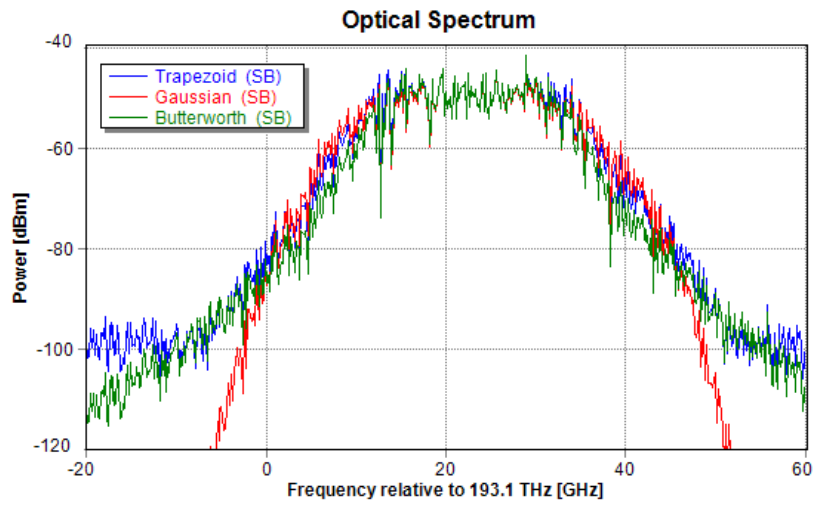

Fig. 5. Optical response of filters (trapezoid, Gaussian, and Butterworth). 


$$
H_{\text {link }}(f)=\cos \left(\frac{\lambda^{2} \cdot D \cdot \pi \cdot f^{2} \cdot L}{c}\right)
$$

where $D$ is the fiber dispersion, $L$ is the fiber length, $f$ is the modulation frequency, $c$ is the light speed in vacuum, $\lambda$ is the operating wavelength. So, periodic reduction of the transfer function is observed.

Additionally, chirped (intensity- and phase-modulated) signal has more spectral components, it has wider optical band. Consequently, the chromatic dispersion limits the system at shorter length. It affects the optimal optical filter parameters. Small filter offset variations around the optimum offset value result in significant signal degradation. In other words, accurate and stable filter tuning is required to assure optimum performance.

\section{Simulation Results}

First, trapezoidal optical filter was applied and short range fiber link was simulated. Figure 6 shows the PM-IM conversion function applying different slope trapezoidal optical filters. The PM-AM conversion produces high pass filter transfer function. The slope of the conversion curve is higher as the slope of the optical filter increases. So, higher discriminator slope implements better conversion. Next, the transfer function of the system was simulated.

Figure 7 represents the transfer function of the system in case of different optical filter slopes (different PM-IM conversion). The modulation bandwidth is increased as the slope of the optical filter increased. The bandwidth roughly goes with the square of the slope of the optical filter (Fig. 8).

The bandwidth improvement is more effective after transmission over long standard single mode fiber. In this case the low-pass response without off-filtering is reduced by the combined effect of chirp and chromatic dispersion (Fig. 9). The effect is similar for SOA based dispersion compensation, where the chirp of SOA produces optical predistortion, which decreases the degradation effect of dispersion [3].

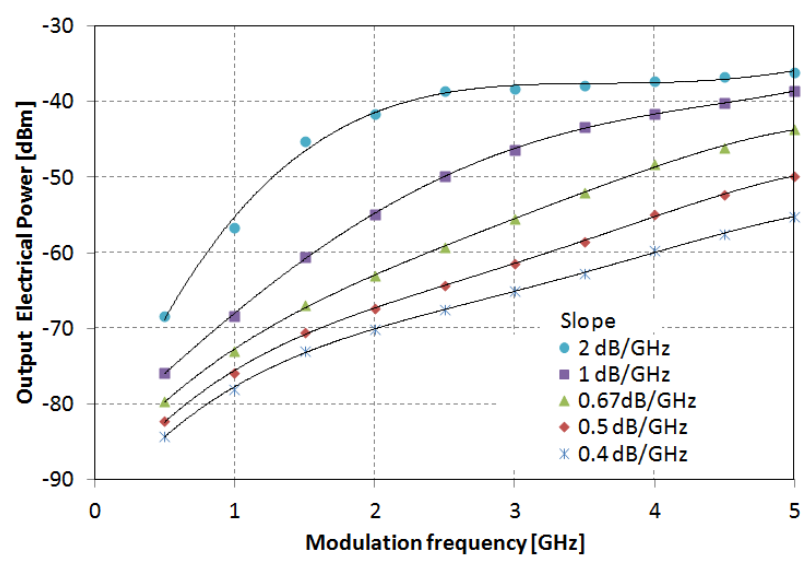

Fig. 6. Frequency function of the PM-IM conversion.

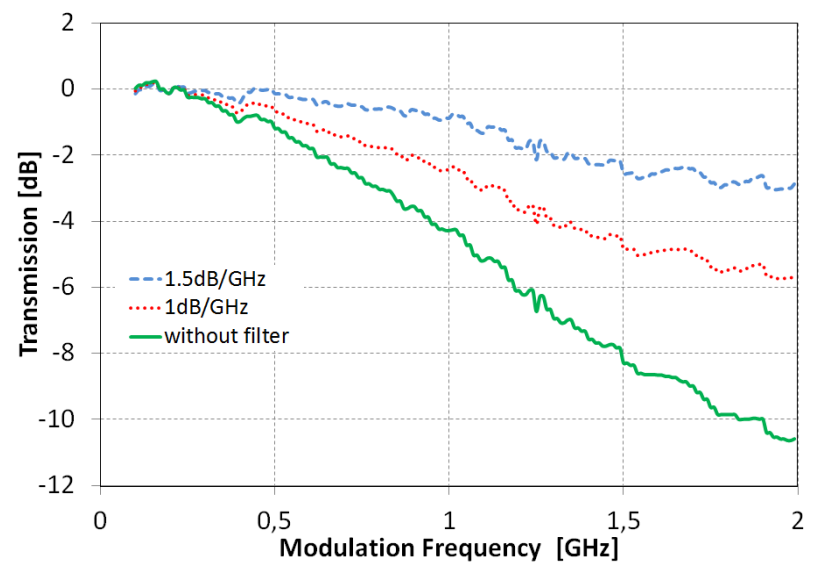

Fig. 7. Transfer function with different optical filter slope.

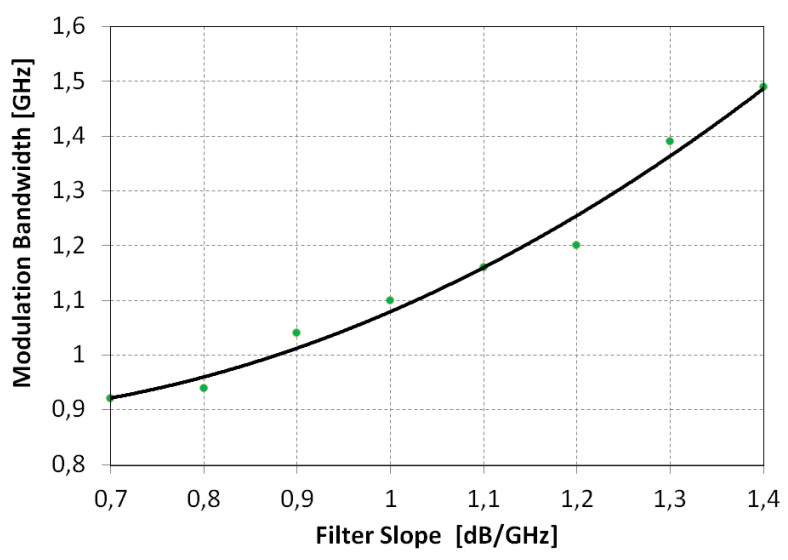

Fig. 8. Modulation bandwidth versus optical filter slope.

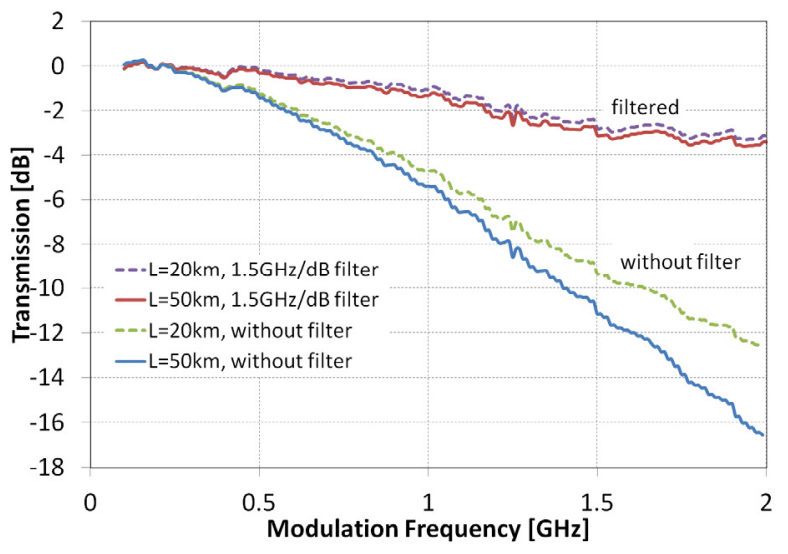

Fig. 9. Transfer function, chromatic dispersion effect.

The improvements of the effective modulation bandwidth as a function of the detuning between the centre frequencies of the optical filter and the optical signal were characterized. Figure 10 represents three detuning cases from no set-off detuning (pure IM) to the maximum allowed detuning. The results validate, that detuning method increases the modulation bandwidth, the optimal detuning value was $23 \mathrm{GHz}$. Extended smooth transmission function provides better bit error rate at high data speed.

Figure 11 compares the efficiency of 3 types (trapezoidal, Gaussian and Butterworth) optical filter applied for 


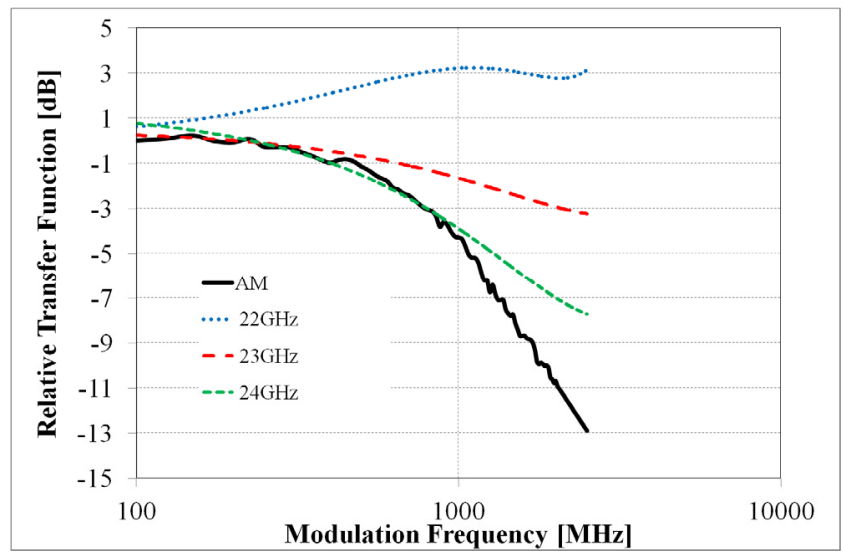

Fig. 10. Relative frequency response of the whole system as a function of the detuning frequency, trapezoidal filter.

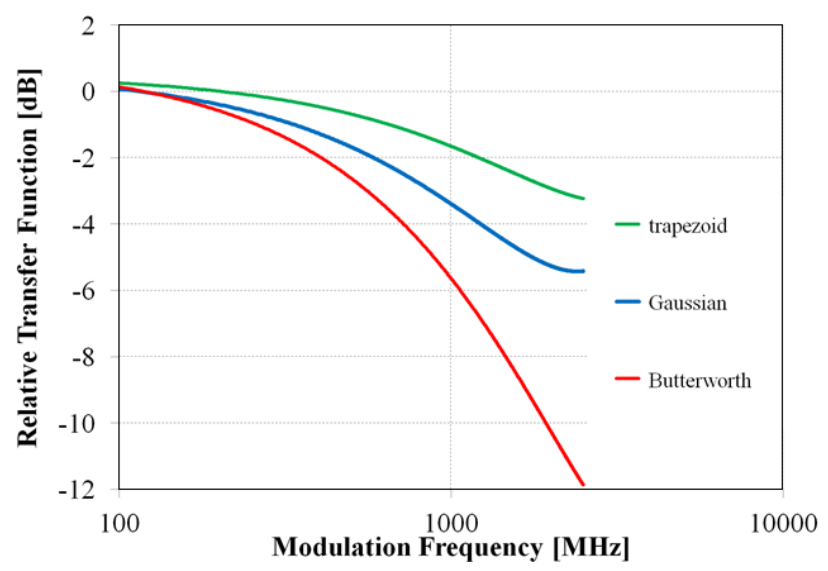

Fig. 11. Relative frequency response of the whole system as a function of the filter.

modulation bandwidth improvement. The simulation results show, that trapezoid filter is the most effective one. The amplitude transfer functions of the three optical filters are similar (Fig. 5), but the phase responses are different. The nonlinear phase responses decrease the efficiency of the bandwidth improvement method.

The figure represents relative frequency responses of the whole system for easier comparison of bandwidth. Another important finding is that higher detuning results in higher insertion loss of the optical filter. The power penalty depends on the slope of the optical filter. However, high slope is necessary for high conversion efficiency. So, it is a trade-off between conversion efficiency and insertion loss.

\section{Conclusion}

This paper has provided a summary of the work, which proves the importance of electrical modulation bandwidth investigation of RSOA based external intensity modulators in next generation optical access networks. Both baseband modulated WDM-PON and high speed WDM-RoF applying Subcarrier Multiplexing systems require high modulation speed. (R)SOA-modulators have several advantages, but the main limitation is the relatively low modulation bandwidth (on the order of $\mathrm{GHz}$ ). Bandwidth enhancement can be achieved by means of optical equalization thanks to the chirping characteristics of RSOA. Direct modulation of RSOA produces optical signal modulated both in amplitude and phase. The limited modulation transfer function can be combined with the converted phase modulation transfer function. The investigation of optical filter with optimal slope for providing a phase-to-amplitude conversion is a crucial challenge.

The paper presented theoretical, simulation and experimental investigation of the proposed approach. The presented simulations describe the slope dependence of the modulation band. The model can take into account the different type of realizable optical filters. However there is a trade-off between modulation efficiency and modulation bandwidth due to the extra insertion loss of the set-off optical filter. The obtained results suggest that the methods enable the use of the RSOA as intensity modulator with improved performance at an extended data rate.

\section{Acknowledgments}

This work was supported by the Hungarian Fund under OTKA No. PD 109288

\section{References}

[1] UDVARY, E., BERCELI, T. Optical subcarrier label swapping by semiconductor optical amplifiers. IEEE Journal of Lightwave Technology, 2003, vol. 21, no. 12, p. 3221-3225. DOI: 10.1109/JLT.2003.819784

[2] UDVARY, E., BERCELI, T. Semiconductor optical amplifier for detection function in radio over fiber systems. IEEE Journal of Lightwave Technology, 2008, vol. 26, no. 8, p. 2563-2570. DOI: 10.1109/JLT.2008.927187

[3] UDVARY, E., BERCELI, T. Combined optical amplification and dispersion compensation in radio over fibre link. IET Optoelectronics, 2010, vol. 4, no. 6, p. 260-266. DOI: 10.1049 /ietopt.2009.0061

[4] UDVARY, E., BERCELI, T. Improvements in the linearity of semiconductor optical amplifiers as external modulators. IEEE Transaction on Microwave Theory and Techniques, 2010, vol. 58, no. 11, p. 3161-6167. DOI: 10.1109/TMTT.2010.2075550

[5] SCHRENK, B., VALICOURT, G., OMELLA, M., LAZARO, J. A., BRENOT, R., PRAT, J. Direct 10-Gb/s modulation of a singlesection RSOA in PONs with high optical budget. IEEE Photonics Technology Letters., 2010, vol. 22, no. 6, p. 392-394. DOI: 10.1109/LPT.2009.2039871

[6] PAPAGIANNAKIS, I., OMELla, M., KLONIDIS, D., et al. Investigation of $10-\mathrm{Gb} / \mathrm{s}$ RSOA-based upstream transmission in WDM-PONs utilizing optical filtering and electronic equalization. IEEE Photonics Technology Letters, 2008, vol. 20, no. 24, p. 2168-2170. DOI: 1109/LPT.2008.2007746

[7] PRESI, M., CHIUCHIARELLI, A., CORSINI, R., CHOUDURY, P., CIARAMELLA, E. Enhanced $10-\mathrm{Gb} / \mathrm{s}$ operation of bandwidth- 
limited R-SOAs without electronic equalization. In Proceedings of the 38th European Conference and Exhibition on Optical Communications (ECOC2012). Amsterdam (Netherlands), 2012, p. 1-3, INSPEC Accession Number: 14028862

[8] OCCHI, L., SCHARES, L., GUEKOS, G. Phase modeling based on the $\alpha$ factor in bulk semiconductor optical amplifiers. IEEE Journal of Selected Topics in Quantum Electronics, 2003, vol. 9, no. 3, p. 788-797, DOI: 10.1109/JSTQE .2003.818346

[9] WATANABE, T., SAKAIDA, N, YASAKA, H. et al. Transmission performance of chirp-controlled signal by using semiconductor optical amplifier. IEEE Journal of Lightwave Technology, 2000, vol. 18 , no. 8, p. 1069-1077. DOI: 10.1109/50.857752

[10] WYRWAS, J. M., WU, M. C. Dynamic range of frequency modulated direct-detection analog fiber optic links. IEEE Journal of Lightwave Technology, 2009, vol. 27, no. 24, p. 5552-5562. DOI: $10.1109 /$ JLT.2009.2031986

[11] SORIN, W. V., CHANG, K.W. Frequency domain analysis of an optical frequency discriminator. IEEE Journal of Lightwave Technology, 1992, vol. 10, no. 6, p. 787-793. DOI: $10.1109 / 50.143079$

\section{About the Author ...}

Eszter UDVARY was born in Budapest, Hungary. She received her Ph.D. degree in Electrical Engineering from Budapest University of Technology and Economics (BME), Budapest, Hungary, in 2009. She is currently an associate professor at BME, Department of Broadband Infocommunications and Electromagnetic Theory, where she leads the Optical and Microwave Telecommunication Lab. Dr. Udvary's research interests are in the broad areas of optical communications, including optical and microwave communication systems, Radio over Fibre systems, optical and microwave interactions and applications of special electro-optical devices. Her special research focuses on multifunctional semiconductor optical amplifier application techniques. She is deeply involved in visible light communication, indoor optical wireless communication and microwave photonics techniques. 